\title{
Tratamiento térmico con láser de cuchillas de acero para uso agrícola ${ }^{(\bullet)}$
}

\author{
G. Muñiz*, A. Conde**, B.J. Fernández**, R. Varela*, I. García** y J. de Damborenea**
}

Resumen

\begin{abstract}
En el presente trabajo se muestran los resultados obtenidos en el temple superficial mediante láser, sobre aceros con distintos contenidos en carbono susceptibles de empleo en la producción de cuchillas de corte para aplicaciones agrícolas. Se comparan los resultados de los distintos aceros en función de su estudio metalográfico, perfiles de dureza y ensayos de resistencia al desgaste y a la corrosión. Destacan los resultados obtenidos con el acero de $0,33 \%$ de carbono, por tratarse de un acero experimental de producción reciente para su empleo en cuchillas de uso agrícola. Tras el estudio metalográfico, se aprecia, en la capa superficial del metal tratado, la existencia de tres zonas bien definidas: la zona tratada en la que ocurren las transformaciones de fase típicas del temple, una zona de transición, que podemos considerar como la zona afectada térmicamente (ZAT), con transformaciones parciales y, por último, el metal base con su microestructura de origen. Las durezas conseguidas, en todos los casos, son muy superiores a las del acero no tratado, en una profundidad de, hasta, $600 \mu \mathrm{m}$. La resistencia al desgaste mejora notablemente con el tratamiento láser, sin que disminuya, por efecto del mismo, la resistencia a la corrosión en medio salino.
\end{abstract}

Palabras clave Endurecimiento superficial con láser. Acerós. Dureza. Desgaste. Corrosión

\section{Laser heat treatment of steel cutting blades for agricultural uses}

\begin{abstract}
The present paper focuses on the laser surface treatment of mild steels with different \%wt of carbon to be used as cutting blades in agricultural applications. The results are discussed in function of the metallographic study, hardness profiles, wear resistance and corrosion testing. Special attention is paid to the results of the results obtained with the experimental steel with carbon $0.33 \%$ wt because it is a new promising material specifically developed to be applied in such agricultural uses. Metallographic studies showed three well defined regions: the laser treated zone, where solid state phase transformation occurs, the heat affected zone (HAZ) with partial transformations and finally the bulk metal with the original microstructure. Surface hardness was in all cases higher than the base steel in a depth range $600 \mu \mathrm{m}$. Wear resistance is notably improved with laser heat treatment while no detrimental effects were induced in their specific corrosion resistance.
\end{abstract}

Keywords Laser heat treatment. Steels. Hardness. Wear. Corrosion

\section{INTRODUCCIÓN}

El empleo de las distintas técnicas láser en tratamientos tecnológicos avanzados, en diferentes ramas de la industria moderna es, hoy en día, una realidad. Estudios recientes sobre el mercado de equipos láser para procesamiento de materiales (medias y altas potencias), en el año 2002, ratifican el uso creciente de este tipo de equipos y, el pronóstico para el año 2003, confirma esta tendencia ascendente ${ }^{[1]}$.
La posibilidad de enfocar un haz láser en áreas específicas de la superficie de un metal permite la realización de tratamientos localizados, con el fin de aumentar alguna de las características del material base. Cuando un haz láser de una potencia dada irradia una superficie metálica con una determinada velocidad de barrido, la energía absorbida sobre la superficie produce un calentamiento continuo de la misma que, en función de los parámetros de procesado elegidos, puede llegar a su fusión.

(•) Trabajo recibido el día 18 de julio de 2003 y aceptado en su forma final el día 5 de noviembre de 2003.

(*) Instituto Superior Politécnico "José A. Echeverría" (ISPJAE). Dpto. de Física. Calle 127 s/n. Aptdo. 6028. Habana 6, Marianao. Ciudad de La Habana.Cuba.E-mail:muniz@electrica.cujae.edu.cu

${ }^{* *}$ Centro Nacional de Investigaciones Metalúrgicas (CENIM/CSIC). Avda. Gregorio del Amo, 8. 28040 Madrid. 
De esta manera, se pueden obtener superficies modificadas mediante la incorporación de nuevos elementos (aleaciones superficiales) o, incluso, desarrollar nuevos recubrimientos (plaqueados) de interés tecnológico ${ }^{[2-4]}$. Igualmente, se pueden generar multicapas o piezas cuasi-finales (prototipado rápido $)^{[5 \text { y } 6]}$.

Un caso particular de los tratamientos con láser, específicamente dentro de la industria mecánica, lo constituye su aplicación en el tratamiento térmico de superficies metálicas, fundamentalmente aceros, con el fin de aumentar la vida útil de piezas que trabajan en condiciones de desgaste y corrosión $^{[7-9]}$. En estos casos, no es necesario alcanzar la temperatura de fusión del material y bastará, en el caso de los aceros, con sobrepasar su temperatura de austenización. Este tipo de tratamiento es, particularmente, interesante en el caso de los aceros con un contenido de carbono suficiente como para producir transformación martensítica. En esta operación de temple superficial se alcanza la temperatura de austenización, en fracciones de segundo. Una vez que el haz se ha desplazado sobre un punto, comienza el ciclo de enfriamiento por conducción a través del resto de la pieza que permanece fría. Naturalmente, factores como la geometría o las propiedades físicas de la pieza, influyen en la velocidad de enfriamiento y, por tanto, en la profundidad de la zona transformada. De esta manera, se consigue obtener superficies duras y resistentes al desgaste y a la corrosión.

En el presente trabajo, se ha procedido a realizar una serie de tratamientos superficiales mediante láser de potencia, sobre tres aceros con diferente contenido en carbono y de interés para su aplicación en cuchillas de corte de uso agrícola. El estudio se centra en el caso de un acero experimental desarrollado en Cuba, con un 0,33 \% de carbono. Tras el tratamiento, se han estudiado los cambios de estructura, microdureza y resistencia al desgaste y a la corrosión de los distintos aceros. Finalmente, se ha empleado un láser de diodos, por las nuevas expectativas que genera su empleo en el tratamiento de materiales metálicos.

\section{PROCEDIMIENTO EXPERIMENTAL}

La composición del acero experimental estudiado, junto a la de los otros empleados como referencia, es la recogida en la tabla I. Igualmente, se ha incluido un acero con un contenido similar en carbono $(0,53 \%)$, al que se ha sometido a un tratamiento mediante láser de diodos, con el fin de
Tabla I. Composición química del acero estudiado

Table I. Chemical composition of the studied steels

\begin{tabular}{ccccccccc}
\hline$C$ & Si & Mn & P & S & Cr & Ni & Mo & $\mathrm{Cu}$ \\
\hline 0,19 & 0,39 & 1,3 & 0,021 & 0,018 & 0,99 & 0,022 & 0,20 & - \\
0,33 & 1,10 & 1,16 & 0,020 & 0,037 & 0,99 & 1,47 & - & - \\
0,61 & 0,31 & 1,1 & 0,021 & $(0,009)$ & 0,21 & 0,19 & $<0,05$ & - \\
0,53 & 0,6 & 0,8 & 0,04 & 0,035 & 1,5 & 0,25 & 0,4 & 0,2 \\
\hline
\end{tabular}

evaluar la capacidad de estos nuevos equipos en el tratamiento de este tipo de materiales.

Las probetas del acero de 0,33 \% de carbono se obtuvieron a partir de palanquillas de una colada experimental, laminadas en caliente hasta un espesor de $12 \mathrm{~mm}$, cuya estructura de partida era bainítica. El resto de materiales se preparó, también, a partir de una chapa laminada del mismo espesor con estructura mixta de ferrita perlita

Los tratamientos láser se realizaron mediante un láser continuo de $\mathrm{CO}_{2}$ con una potencia de salida variable de, hasta, $1.500 \mathrm{~W}$. El haz formado por el sistema óptico fue dirigido, perpendicularmente, sobre la superficie de la probeta, utilizando argón o nitrógeno como gas de protección para evitar la oxidación de las piezas y proteger la óptica del láser. Los tratamientos se llevaron a cabo con potencias nominales de salida de 1.200 y 1.500 W y con velocidades de barrido de $5,8,15$ y 30 $\mathrm{mm} / \mathrm{s}$, con el fin de optimizar las mejores condiciones de tratamiento. Las probetas investigadas se situaron fuera del punto focal para conseguir distintos diámetros del haz sobre la pieza, variables entre 1,2 y $3 \mathrm{~mm}$. En todos los casos y por ser las superficies metálicas muy reflectantes para la longitud de onda del láser de $\mathrm{CO}_{2}$, se utilizó una pintura de grafito coloidal con el fin de incrementar su absorción superficial. Dado el reducido diámetro del haz sobre la pieza fue necesario realizar sucesivas pasadas solapadas $(5,25,50$ y $75 \%$ de solapamiento). Las densidades de potencia obtenidas, entre $10^{3} \mathrm{y}$ $10^{5} \mathrm{w} / \mathrm{cm}^{2}$, se sitúan dentro de los valores críticos recogidos en la bibliografía para tratamientos de temple superficial y fusión ${ }^{[10]}$. Para mayor claridad, los parámetros del tratamiento láser aplicados sobre cada uno de los aceros estudiados se han recogido en la tabla II.

Una vez realizados los distintos tratamientos, se procedió a su estudio metalográfico y al análisis de los perfiles de dureza obtenidos en función del tipo de acero. Paralelamente, se realizaron ensayos de desgaste en seco mediante la técnica de "bloque 
Tratamiento térmico con láser de cuchillas de acero para uso agrícola G. Muñiz, A. CONDE, B.J. FERNÁNDEZ, R. VARELA, I. GARCía y J. DE DAMBORENEA

Tabla II. Parámetros del tratamiento superficial con láser

Table II. Laser parameters used to carry out surface treatments

\begin{tabular}{lccc}
\hline \% C del acero. Parámetros & 0,33 & 0,19 y 0,61 & 0,53 \\
\hline Potencia nominal máxima $(\mathrm{W})$ & 1.200 & 1.500 & 3.000 \\
Diámetro del haz $(\mathrm{mm})$ & $1,2-2,8$ & 3 & 22 \\
Velocidad de proceso $(\mathrm{mm} / \mathrm{s})$ & 5,8 y 15 & 15 y 30 & 6 \\
Densidad de potencia $\left(\mathrm{W} / \mathrm{cm}^{2}\right)$ & $1,0^{6} \cdot 10^{5}-$ & $21,2 \cdot 10^{3}$ & 789 \\
& $19,5 \cdot 10^{3}$ & & \\
\hline
\end{tabular}

sobre anillo", utilizando un anillo de acero SAE 10 (dureza 63HRC) y de $35 \mathrm{~mm}$ de diámetro, a temperatura ambiente y una humedad del $50 \%$. Los ensayos se realizaron a 100 r.p.m., con una carga aplicada de $10 \mathrm{~N}$, determinándose la anchura de la huella de acuerdo a la norma ASTM G77-93.

Finalmente, los estudios para evaluar su comportamiento frente a la corrosión se llevaron a cabo mediante la realización de ensayos electroquímicos de corriente continua, más concretamente, de curvas de polarización sobre las zonas tratadas con láser. Para ello, se utilizó un montaje clásico de tres electrodos, constituido por un electrodo de referencia de calomelanos saturado, un contraelectrodo de platino y $1 \mathrm{~cm}^{2}$ de la superficie tratada, que constituyó el electrodo de trabajo. Estos ensayos consistieron en un barrido de potencial a velocidades muy lentas, $0,16 \mathrm{mV} / \mathrm{s}$, y en sentido anódico, desde un valor inicial de potencial, localizado por debajo del potencial de circuito abierto. De esta forma, se obtienen las ramas catódica y anódica, respectivamente, de la curva de polarización. Una vez que la densidad de corriente alcanza valores elevados, del orden de $0,250 \mathrm{~mA} / \mathrm{cm}^{2}$, se inicia le barrido en sentido inverso, con el fin de estudiar la reversibilidad del sistema y la probabilidad de repasivación de las picaduras formadas, en el caso en qué estas se hubieran producido.

\section{RESULTADOS Y DISCUSIÓN}

En la figura 1 se presenta una macrografía de la forma de la cuchilla de corte con las huellas correspondientes a las zonas tratadas. Las probetas irradiadas se prepararon para su estudio metalográfico, cortándolas en un plano perpendicular a la dirección del tratamiento, se pulieron y se atacaron químicamente con Nital $2 \%$. En la figura 2 se presenta una micrografía representativa de la zona tratada, correspondiente al acero experimental de

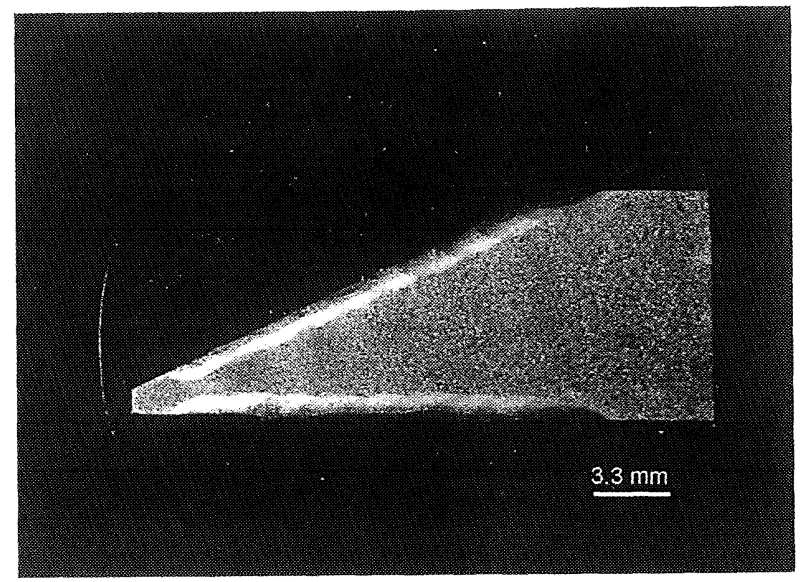

Figura 1. Macrografía de las cuchillas y de las zonas tratadas.

Figure 1. Macrograph of the cutting blades and treated zones.

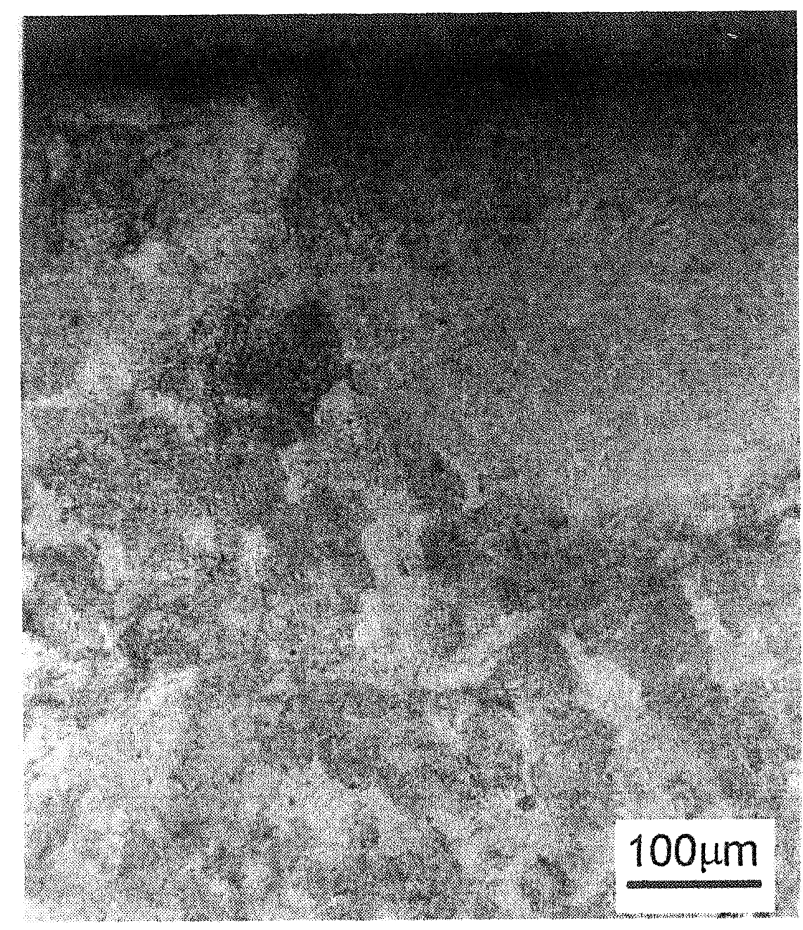

Figura 2. Micrografía de la zona afectada por el calor. Acero con $0,33 \%$ de carbono. $\varnothing=1,2 \mathrm{~mm} ; \mathrm{v}=5 \mathrm{~mm} / \mathrm{s}$, potencia de salida $=1.200 \mathrm{~W}$.

Figure 2. Micrograph of the heat affected zone. Steel with carbon $0.33 \% \varnothing=1.2 \mathrm{~mm} ; \mathrm{v}=5 \mathrm{~mm} / \mathrm{s}$; Output power = $1200 \mathrm{~W}$.

0,33 \% de carbono. En todos los casos (independientemente del tipo de acero y de los parámetros del procesado), se alcanzó la temperatura de austenización. La imagen corresponde a una probeta tratada con una densidad de energía de, aproximadamente, $3.000 \mathrm{~J} \cdot \mathrm{cm}^{-2}$. 
Tratamiento térmico con láser de cuchillas de acero para uso agrícola G. Muñiz, A. Conde, B.J. Fernández, R. VArela, I. García y J. DE DAmborenea

Se desecharon las condiciones de tratamiento en las que se produce fusión de la superficie del material. Esta situación no es la más deseable por doble motivo: porque la presencia de zonas fundidas en el material altera la rugosidad de la superficie y obliga a un rectificado posterior, y porque en las zonas de filo puede producirse un calentamiento tan elevado que no se den las condiciones necesarias para conseguir una estructura típica de temple. Mediante el corte transversal de la muestra y su inspección metalográfica se aprecia la presencia de estructuras procedentes de la fusión del material o de las correspondientes a un temple inadecuado. En este punto, deberíamos destacar que la aplicación de métodos analíticos de modelización del proceso o la monitorización directa del mismo, podría ayudar a evitar este tipo de problemas, como ha quedado reflejado en algunos trabajos aparecidos en la bibliografía ${ }^{[11-13]}$.

En las condiciones de trabajo seleccionadas se superó la temperatura de austenización del acero estudiado y, dadas las altas velocidades de enfriamiento, se produjo la transformación martensítica. Esta estructura de temple es más apreciable en las proximidades de la superficie. A mayor profundidad puede distinguirse la zona afectada, con austenización parcial, donde no se consiguió una transformación total a martensita. Finalmente, nos encontramos con la microestructura de partida del material base. Aunque, como se ha comentado, en todos los aceros estudiados se consiguió la austenización y posterior transformación en martensita de la capa tratada, en el acero de bajo contenido en C $(0,19 \%)$, esta transformación es menor que en los otros casos estudiados.

Desde el punto de vista de la utilización de este tipo de materiales, la zona en la que se ha realizado una transformación total es la que, realmente, interesa. En la figura 3 se presenta un detalle de dicha estructura martensítica. Esta microestructura procede de una zona completamente templada del material austenizado, junto a una mezcla de estructuras correspondientes a las zonas parcialmente austenizadas. Dada la similitud de los tres aceros al carbono, las diferencias microestructurales entre estos tres aceros son muy bajas y residen, fundamentalmente, en la dureza de la martensita obtenida y en las profundidades de temple alcanzadas para cada una de ellas.

Como consecuencia de este cambio de estructura, las propiedades mecánicas del material, más concretamente la dureza, también, experimentan cambios significativos. En la figura 4, se presentan

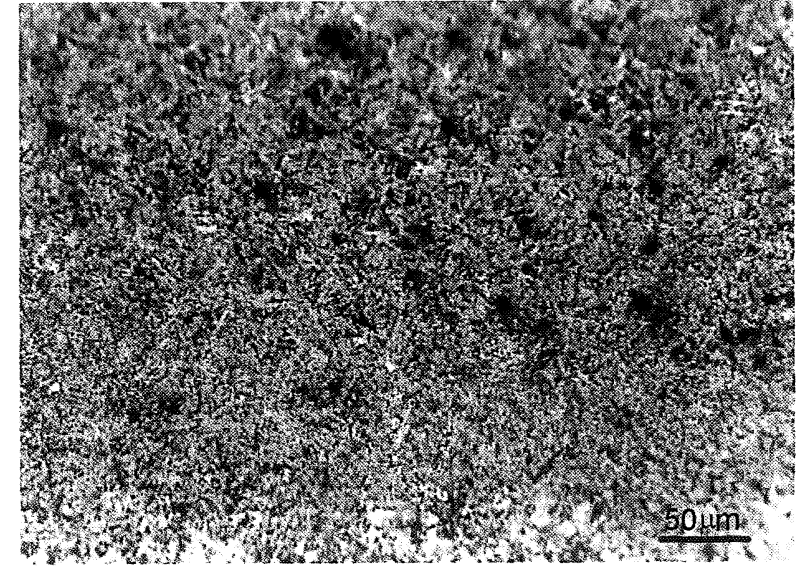

Figura 3. Micrografía de la estructura martensítica en la zona afectada por el calor del acero con 0,33 \% de carbono.

Figure 3. Micrograph of the martensitic structure in the heat affected zone for steel with carbon $0.33 \%$.

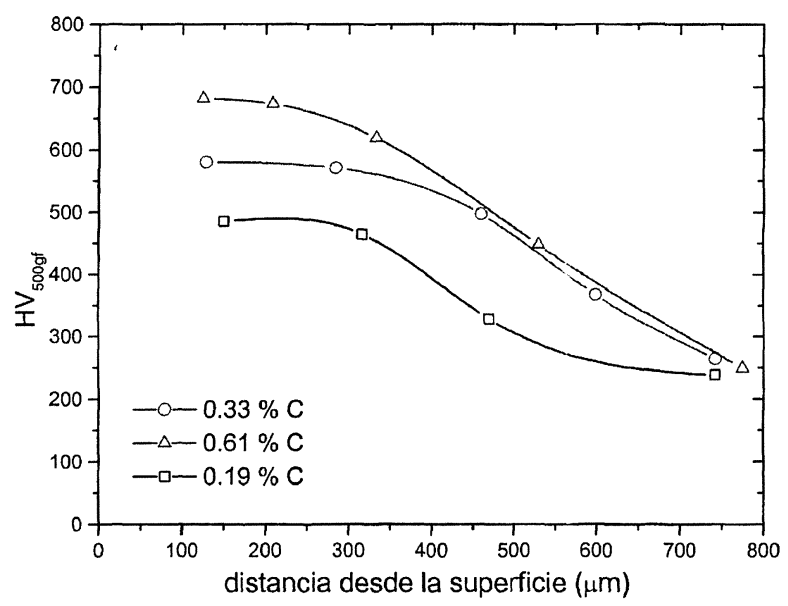

Figura 4. Perfiles de dureza en profundidad para los aceros estudiados.

Figure 4. Hardness depth profiles corresponding to the studied steels.

los perfiles de dureza en función del contenido en carbono de cada uno de los aceros tratados. En ella, se aprecia que los valores de dureza alcanzados en la zona tratada, en todos los casos, duplica a las del material base. Además, la profundidad del temple alcanzado es de unos $300 \mu \mathrm{m}$ pero, como puede deducirse de la pendiente de las curvas, la zona afectada térmicamente es, considerablemente, mayor.

Con respecto a la influencia del solape en las variaciones de dureza, en la figura 5 se presenta la variación de dicho parámetro realizada $100 \mu \mathrm{m}$ por debajo de la superficie y a lo largo de varios cordones solapados $(25 \%)$. Este solape se ha elegido porque permite atenuar el efecto del revenido sobre los trazos anteriores. Se aprecia, claramente, 
Tratamiento térmico con láser de cuchillas de acero para uso agrícola G. MuñIz, A. CONDE, B.J. FERNÁNDEZ, R. VARELA, I. GARCÍA y J. DE DAMBORENEA

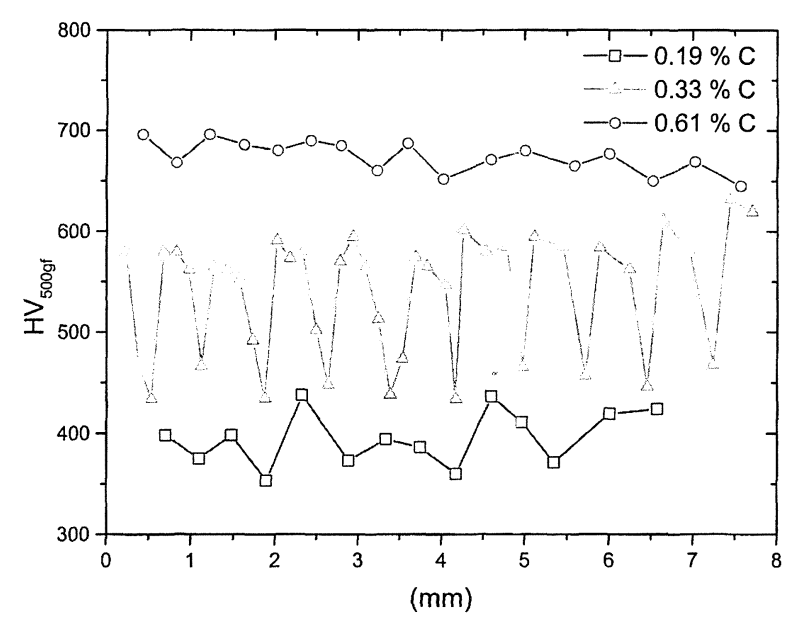

Figura 5. Perfiles de dureza paralelos a la superficie medidos a $100 \mu \mathrm{m}$ de profundidad sobre varios cordones solapados.

Figure 5. Hardness profiles measured $100 \mu \mathrm{m}$ above the surface across some overlapping tracks.

para los aceros de 0,19 y 0,33 \% de carbono, la caída de la dureza en las zonas correspondientes al solape, por efecto del revenido provocado por el segundo tratamiento térmico que produce la segunda pasada del haz láser. Las condiciones representadas para el acero de 0,61\% de carbono con pequeño solape permiten que, en la zona estudiada, se produzca un menor efecto de revenido, lo que supone una ventaja.

Dado el posible interés de estos tratamientos para el aumento de la vida útil de las cuchillas de corte de uso agrícola, se procedió a evaluar su resistencia frente al desgaste. Para ello, se prepararon bloques paralelepipédicos, una de cuyas caras, la que estaba en contacto con el anillo, estaba tratada con láser. En la figura 6 se presenta la evolución de la pérdida de masa con la distancia deslizada. Como puede apreciarse, en los 300 primeros metros deslizados, todas las probetas presentan un aumento lineal del volumen desgastado. El acero con $0,61 \%$ de carbono presenta una buena resistencia al desgaste, incluso antes del tratamiento con láser, en correspondencia con su estructura de perlita con alto contenido en carbono, que es muy adecuada para resistir estos fenómenos. No puede decirse lo mismo del acero de 0,19 \% de carbono que, aún después del tratamiento láser, presenta valores de desgaste superiores a los de los dos aceros restantes antes de someterse al mismo. El acero experimental presenta una disminución del volumen de masa perdida por desgaste, de $0,5 \mathrm{~mm}^{3}$, con relación al valor correspondiente al acero virgen, lo que puede considerarse como satisfactorio.

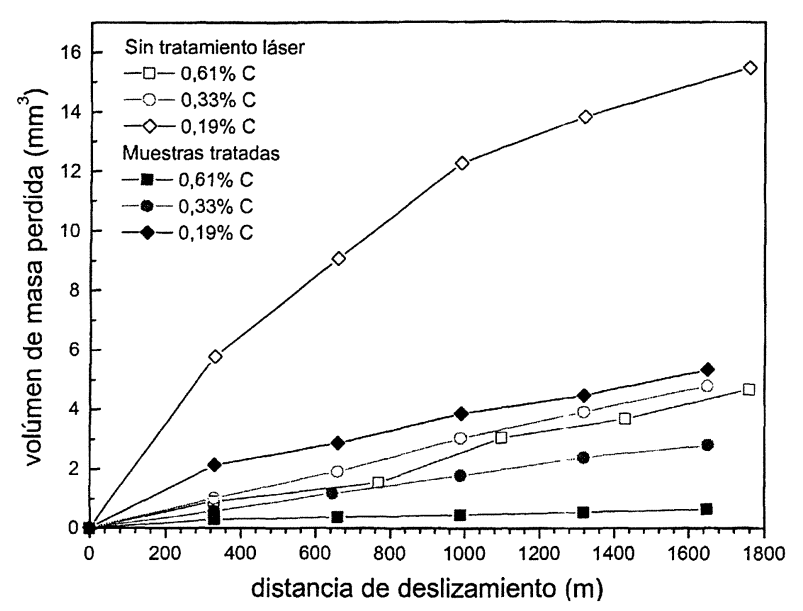

Figura 6. Pérdida de masa con la distancia deslizada. Ensayo de bloque sobre anillo; carga $=10 \mathrm{~N}, 100$ r.p.m.

Figure 6. Mass loss with sliding distance. Block on ring test; load $=10 \mathrm{~N}, 100$ r.p.m.

Aunque, evidentemente, el comportamiento al desgaste del acero de 0,61\% de carbono es mucho mejor, este acero puede presentar problemas en el procesado de la zona afilada ya que, para las mismas condiciones de procesado, las profundidades de temple son mayores y esto reduce su resistencia al choque, en la aplicación que se desea desarrollar. Es importante tener en cuenta que, en el campo en el que se recolecta la caña de azúcar, pueden existir piedras o restos metálicos procedentes de otras campañas anteriores que pueden causar la rotura de las cuchillas por impacto.

En los tres casos, el tratamiento láser mejora la resistencia al desgaste reduciendo la cantidad de volumen de material desgastado por unidad de longitud deslizada. A partir de dichos datos, se evaluó la velocidad de desgaste definida como el volumen de material desgastado por unidad de longitud deslizada y fuerza aplicada $\left(\mathrm{mm}^{3} / \mathrm{Nm}\right)$, obteniéndose una disminución de, casi, un orden de magnitud para la velocidad de desgaste del acero experimental.

Paralelamente, las cuchillas de referencia, con aceros de 0,61 y $0,19 \%$ de carbono con tratamiento térmico láser, se sometieron a ensayos de campo en máquinas cosechadoras de caña de azúcar, en suelos con alto contenido de sílice, pudiendo apreciarse en la figura 7 el desgaste experimentado en el caso de 0,61 \% de carbono. Durante su vida en servicio, se consigue un comportamiento satisfactorio del material, el cual revela un desgaste uniforme, libre de roturas por choque, a pesar de que, ésta, es una de las causas más habituales que provocan la sustitución de las cuchillas. En la actualidad, las cuchillas fabricadas a partir del acero 

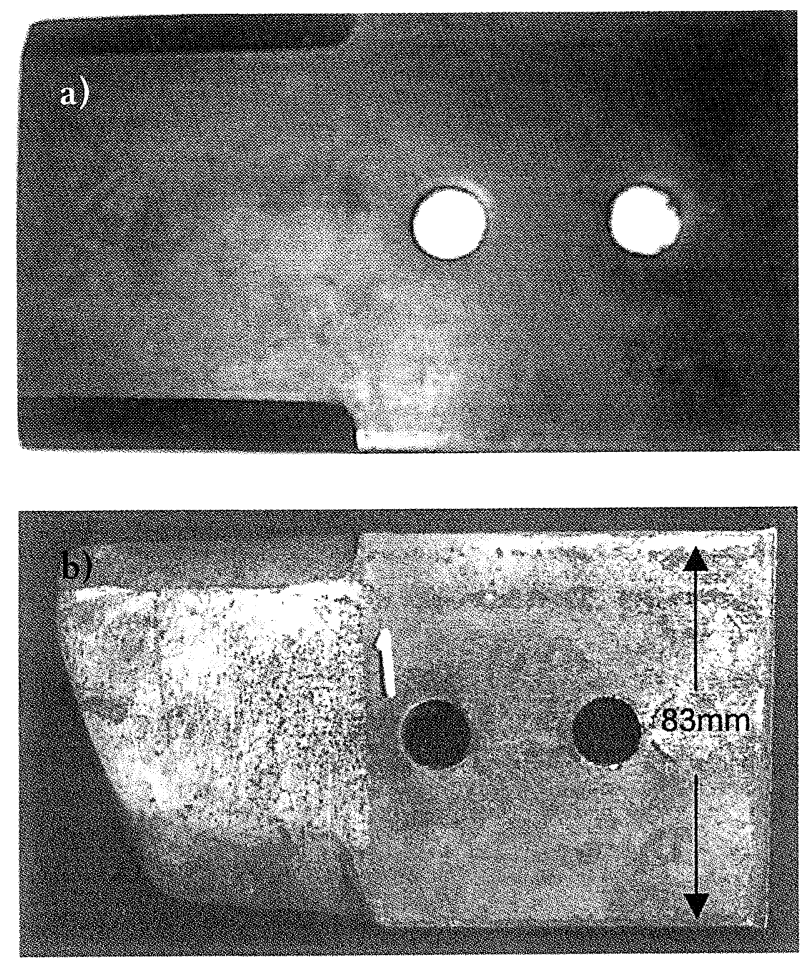

Figura 7. Cuchilla de acero con 0,61\% de carbono antes y después de la prueba de campo.

Figure 7. Carbon steel blade (carbon $0.61 \%$ ) before and affer service work

experimental con 0,33 \% de carbono, se encuentran en fase de prueba en campo.

Como se ha podido ver en la figura 5 , uno de los inconvenientes que presenta el tratamiento con láser es que, debido al pequeño diámetro del haz láser empleado, se hace necesario realizar sucesivas pasadas para tratar el filo de la cuchilla, lo que origina diferentes estructuras y durezas a lo largo del mismo. Para evitar esto y con el fin de optimizar el proceso desde un punto de vista económico, se procedió al estudio de la microestructura obtenida en un acero de características similares $(0,53 \%$ de carbono; véase tabla I), mediante un láser de diodos de $3 \mathrm{~kW}$ de potencia (Tabla II), con el fin de evaluar las posibles aplicaciones de este tipo de láser en el tratamiento de cuchillas de uso agrícola. La utilidad de este nuevo tipo de láser está evaluándose, actualmente, como alternativa a los equipos convencionales ${ }^{[14 \mathrm{y} 15]}$. Hay que resaltar, como ventaja añadida, que debido a su longitud de onda en torno a los $800 \mathrm{~nm}$, no se necesita emplear recubrimientos absorbentes (tipo pinturas de grafito). En este caso, los cordones obtenidos son homogéneos, con un ancho de $22 \mathrm{~mm}$ y una profundidad de $1,12 \mathrm{~mm}$. En la macrografía de la figura 8 se puede apreciar el rastro obtenido, destacán-

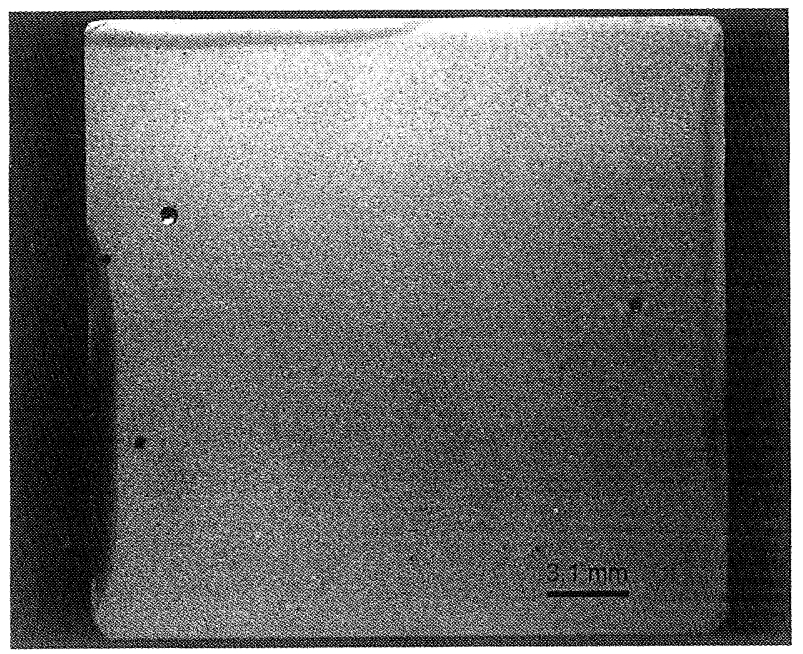

Figura 8. Cordones láser obtenidos mediante láser de diodos en un acero de 0,53\% de carbono.

Figure 8. Laser tracks obtained by means a diode laser on carbon steel of carbon $0.53 \%$.

dose el ancho logrado en una sola pasada, que sería suficiente como tratar todo el filo de una sola vez. Además y debido al relativo bajo aporte energético, $789 \mathrm{~W} \cdot \mathrm{cm}^{-2}$, sólo se produce el calentamiento suficiente como para austenizar el material, sin los problemas derivados de la fusión, descritos anteriormente. En la figura 9 se presenta la estructura martensítica obtenida. En la figura 10 pueden apreciarse los valores de dureza alcanzados con los dos tipos de tratamientos con láser en los aceros con contenido de carbono superior a 0,5\%. En ella, podemos ver que la variación de la dureza con la distancia a la superficie es la correspondiente a

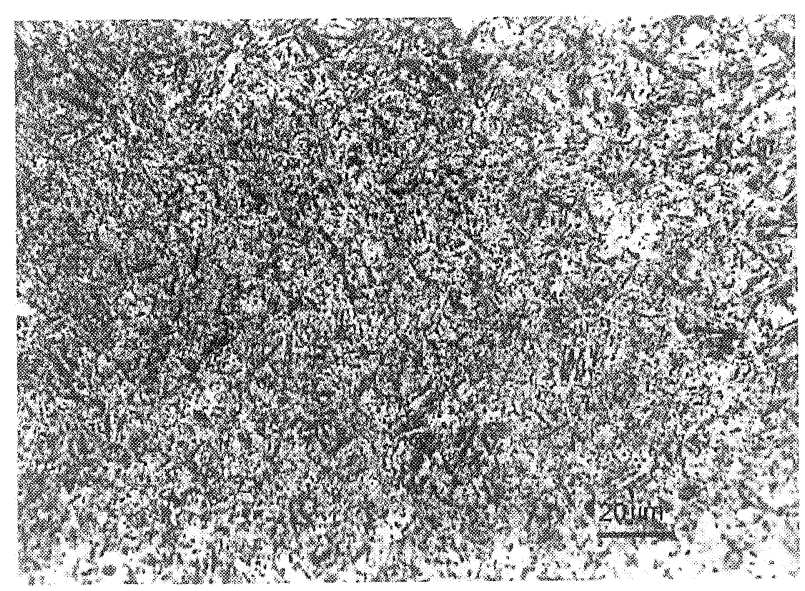

Figura 9. Estructura martensítica obtenida mediante el tratamiento superficial con láser de diodos en un acero con $0,53 \%$ de carbono.

Figure 9. Martensitic structure induced on a steel with carbon $0.53 \%$ by a diode laser surface treatment.

Rev. Metal. Madrid 39 (2003) 443-451 


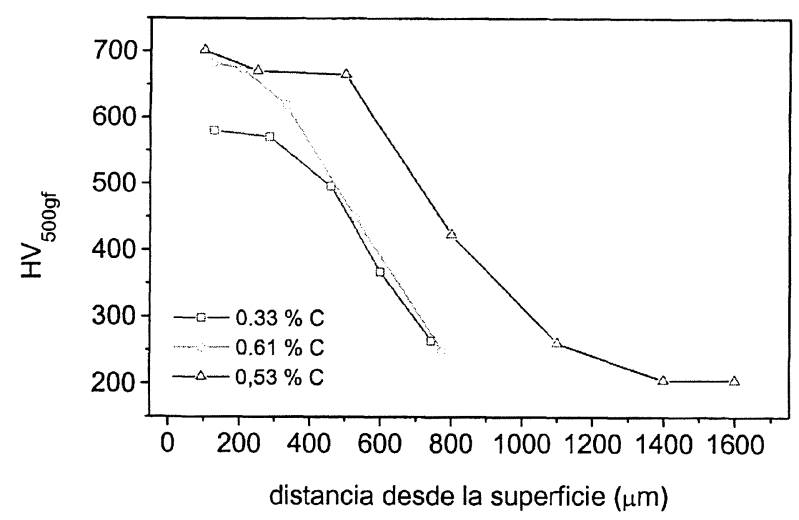

Figura 10. Comparación de la dureza alcanzada mediante el láser de diodos para un acero con contenido intermedio de carbono respecto de los aceros tratados con un láser de $\mathrm{CO}_{2}$.

Figure 10. Comparison of hardness values obtained for a diode laser treated steel with intermediate carbon content with respect to the steels treated with $\mathrm{CO}_{2}$ laser.

un temple superficial. La dureza alcanzada en la superficie aumenta con el contenido de carbono y, en ambos casos, se consiguen profundidades de temple suficientes como para garantizar un buen comportamiento en servicio.

Finalmente, uno de los aspectos que debe tenerse en cuenta a la hora de decidirse sobre este tipo de tratamientos es, que no resulten prejudiciales para otras propiedades, como es el caso de la corrosión. Si bien, estos aceros no son especialmente recomendables desde el punto de vista de la corrosión, si debemos tener en cuenta que, debido al propio ambiente de corte, la corrosión debe mantenerse por debajo de un mínimo aceptable. Por esta razón, se procedió a la realización de ensayos electroquímicos sobre las muestras tratadas en soluciones, con una muy baja concentración de cloruros, con el fin de establecer los posibles cambios introducidos por el tratamiento láser en el comportamiento frente a la corrosión. De manera general, se puede afirmar que el tratamiento láser no introduce cambios notables en ninguno de los aceros, manteniéndose los niveles de resistencia a la corrosión del mismo orden con respecto del material sin tratar. La forma de las curvas de polarización es la misma, antes y después del tratamiento superficial, indicando que la velocidad de corrosión se mantiene dentro del mismo rango.

Para las muestras con un mayor contenido en C, 0,61 \% en masa, el mecanismo de corrosión es el de un control mixto del proceso, caracterizado por una velocidad de corrosión del orden de $10^{-6} \mathrm{~A} / \mathrm{cm}^{2}$ (Fig. 11). Por el contrario, las muestras

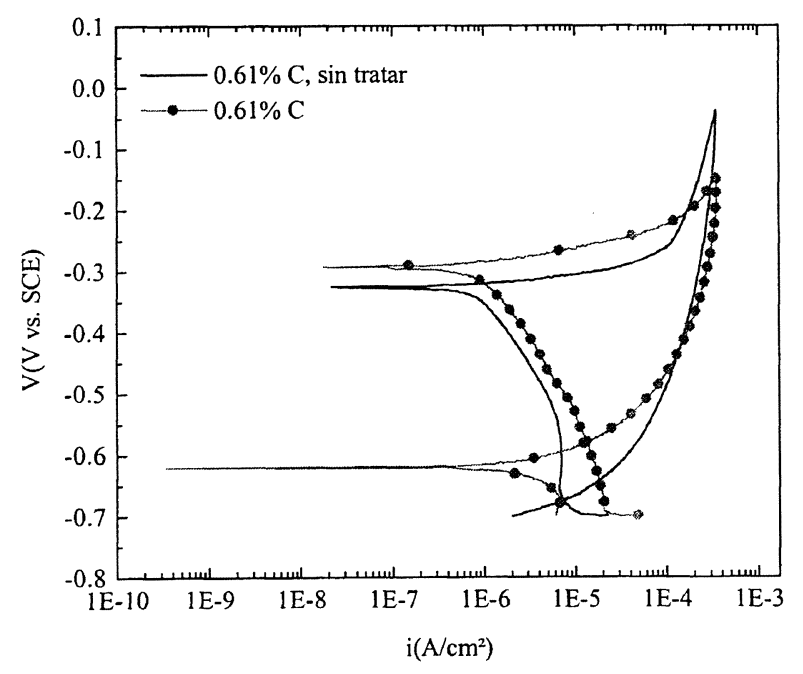

Figura 11. Curvas de polarización en $\mathrm{NaCl} 0,003 \mathrm{M}$ correspondiente al acero con $0,61 \%$ de carbono.

Figure 11. Polarization curves in $\mathrm{NaCl} 0.003 \mathrm{M}$ corresponding to steel with carbon $0.61 \%$.

correspondientes al acero experimental (Fig. 12), presentan un control por difusión del proceso, caracterizado por una velocidad límite de difusión de $2 \cdot 10^{-5} \mathrm{~A} / \mathrm{cm}^{2}$. Tras el tratamiento láser, se produce una disminución de la densidad de corriente límite hasta valores de $7 \cdot 10^{-6} \mathrm{~A} / \mathrm{cm}^{2}$, que indican un atenuamiento de la velocidad de disolución del material. Finalmente, el substrato de menor contenido en carbono (Fig. 13), revela un cambio en el proceso con el tratamiento, que pasa de ser mixto a estar controlado con difusión. No obstante, pese a este ligero cambio en la forma en la curva, la

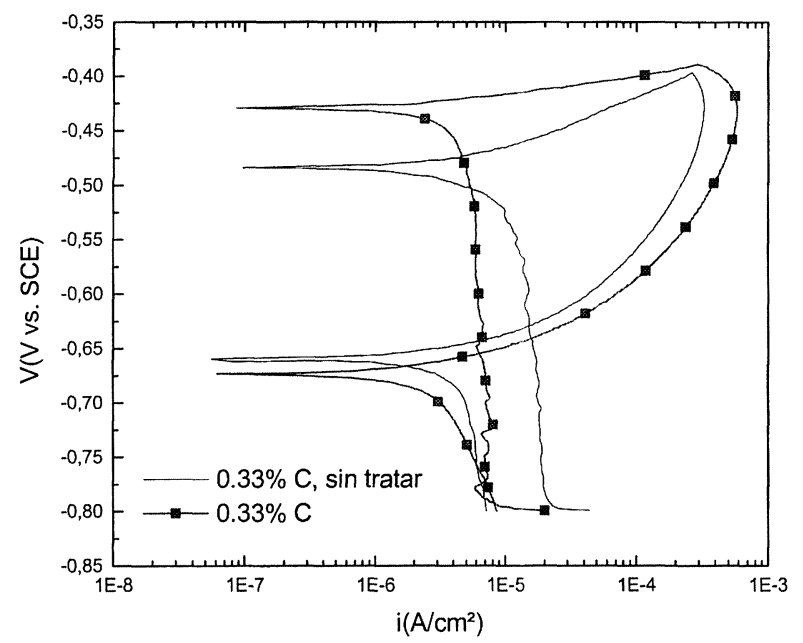

Figura 12. Curvas de polarización en $\mathrm{NaCl} 0,003 \mathrm{M}$ correspondiente al acero con $0,33 \%$ de carbono.

Figure 12. Polarization curves in $\mathrm{NaCl} 0.003 \mathrm{M}$ corresponding to steel with carbon $0.33 \%$. 


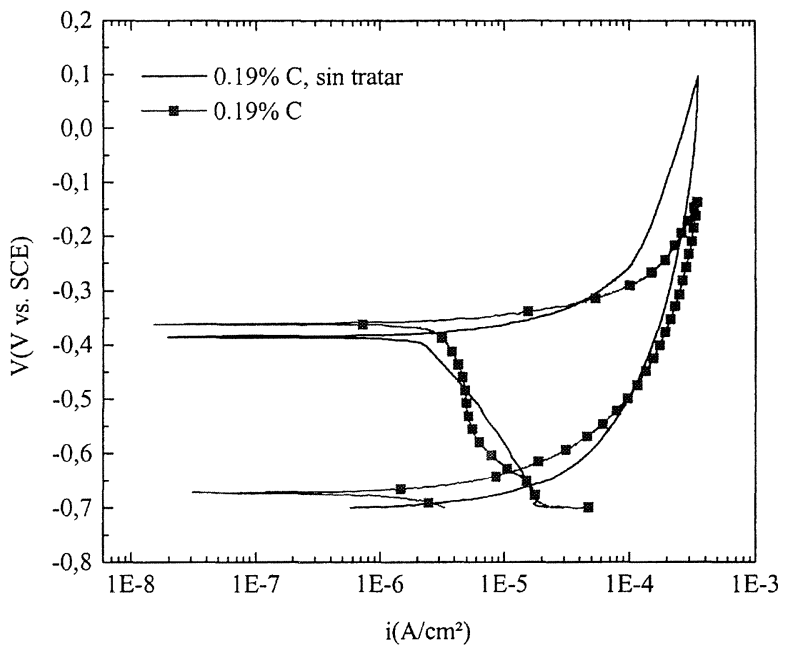

Figura 13. Curvas de polarización en $\mathrm{NaCl} 0,003 \mathrm{M}$ correspondiente al acero con $0,19 \%$ de carbono.

Figure 13. Polarization curves in $\mathrm{NaCl} 0.003 \mathrm{M}$ corresponding to steel with carbon $0.19 \%$.

velocidad de disolución del metal es del mismo orden, $10^{-6} \mathrm{~A} / \mathrm{cm}^{2}$, antes y después del tratamiento.

Por otra parte, el potencial de corrosión se desplaza ligeramente hacia valores más nobles. Estos cambios en la dirección más noble parecen estar relacionados con el contenido de carbono en la composición del acero y con las condiciones de procesado del láser. Al igual que sucedía con la densidad de corriente, los incrementos de potencial son más acusados para el caso del substrato del acero experimental con un $0,33 \%$ de carbono.

En principio, aunque estas variaciones observadas no suponen un notable aumento de la resistencia frente a la corrosión, el tratamiento láser tampoco introduce un empeoramiento del comportamiento.

\section{CONCLUSIONES}

- La respuesta al tratamiento térmico láser del acero experimental es notable, aumentada por el alto contenido en níquel de dicho acero. Los resultados logrados demuestran la posibilidad del uso de la técnica láser en este tipo de aceros, para incrementar su dureza superficial y su resistencia al desgaste. La presencia de elementos de aleación y las altas velocidades de calentamiento y enfriamiento del material que se obtienen con el empleo del láser, favorecen la formación de las microestructuras duras, características del acero templado.

La microestructura de la superficie de las muestras tratadas cambia a una martensita fina, existiendo una zona afectada térmicamente con una microestructura mixta. La dureza superficial de las probetas se incrementa después del tratamiento térmico láser. El solape crea zonas superficiales heterogéneas, debido al revenido de la martensita en la zona recalentada, que no representan problema desde el punto de vista de desgaste.

- El ensayo de desgaste revela el incremento de la resistencia al desgaste de las probetas tratadas, en comparación con las no tratadas, en los tres aceros considerados.

- Por otra parte, los ensayos electroquímicos indicaron que, si bien no se produce una mejora del comportamiento respecto del material base, derivada de la heterogeneidad estructural obtenida por el solapamiento de los sucesivos cordones, tampoco se introduce un empeoramiento del comportamiento del material.

- Finalmente, hay que destacar que, el tratamiento superficial con láser es adecuado para este tipo de aceros y, esto, permite creer que el empleo de láseres de diodos es, especialmente interesante por su versatilidad, absorción y tamaño del haz, ya que permitiría obtener cordones con anchos similares al filo de la cuchilla, reduciendo el tiempo de tratamiento de cada una de ellas y aumentando la eficiencia del proceso.

\section{Agradecimientos}

Este trabajo ha sido parcialmente financiado en su parte experimental por el proyecto FEDER 2FD1997-2020-C03-02 y por el proyecto de cooperación CSIC/CITMA 2003 CU0017 que ha financiado las estancias de los investigadores en ambos países.

Los autores quieren agradecer al Prof. Rui Vilar del Instituto Superior Técnico de Lisboa y a Fidel Zubiri de CETENASA los tratamientos con láser realizados en sus instalaciones.

\section{REFERENCIAS}

[1] K. Kinkade, S.G. Anderson y R. Steele, Laser Focus World 39 1-2 (2003) 73-98.

[2] G. Thawari, G. Sundarararjan y DS.V. Joshi, Thin Solid Films 423 (2003) 41-53.

[3] M.H. Staia, M. Cruz y Narendra B. Dahore, Thin Solid Films 377-378 (2000) 665-674; J. Mater. Process. Tech. 122 (2002) 63-68. 
[4] J. DE Damborenea, Surf. Coat. Technol. 100-101 (1998)377-382.

[5] Y.P. Kathuria, Surf. Coat. Technol. 116-119 (1999) 643-647

[6] J. Laeng, J.G. Stewart y F.W. Liou, Int. J. Prod. Res. 38 (2000) 3.973-3.996.

[7] J.C. Ion, Surf. Eng. 18 (2002)14-31.

[8] A.M. Prokhorov, V.I. Konov, I. Ursu y I.N. MiHAILESCU, Laser Heating of metals, Adam Hilger, Bristol, 1990.

[9] D.I. Pantelis, E. Bouyiouri, N. Kouloumbi, P. Vassiliou y A. Koutsomichalis, Surf. Coat. Technol. 298 (2002) 125-134.
[11] RyKalin y Uglov, Laser and electron beam Handbook, Editorial Mir, 1985.

[12] J. Ruiz, B.J. Fernández, J.M. Belló, J.M. SAnz y F. Zubiri, Rev. Metal. Madrid 26 (1990) 374-385.

[13] A. Yánez, J.C. Álvarez, A.J. López, G. Nicolás, J.A. Pérez, A. Ramil y E. SaAvedra, Appl. Surf. Sci 186 (2002) 611-616.

[14] A. Molpeceres, R. Catalina y J.L. Ocaña, Rev. Metal. Madrid 38 (2002)195-204.

[15] W. Schulz y R. Poprawe, IEE J. Sel. Top. Quantum Electron. 6 (2000) 696-705.

[16] A. Conde, F.Zubiri y J. DE Damborenea, Mater. Sci. Eng. A334 (2002) 233-238. 